\title{
Revisiones de seguridad basadas en la conducta
}

\section{BBS: Behaviour based on Safety Reviews}

\author{
Tomás Ramos', Alberto Fumanal', David Villarmín' \\ 1. ArcelorMittal Tailored Blanks Zaragoza S.L. España \\ Tomás Ramos \\ Plant Manager \\ ArcelorMittal Tailored Blanks Zaragoza SL \\ Pol. Ind. El Pradillo II parc.9 C/ General Motors, 7 \\ 50690 PEDROLA (Zaragoza) SPAIN \\ $\mathrm{T}+34976613000$ \\ $F+34976612782$ \\ E-mail: tomas.ramos@arcelormittal.com
}

Resumen

Las Revisiones de seguridad basadas en la conducta (BBS: Behaviour based on Safety Reviews) son una herramienta preventiva que ayuda a identificar las conductas inseguras antes de que lleguen a tener lugar cualquier tipo de incidente. Con su práctica diaria en ArcelorMittal Tailored Blanks Zaragoza se promueve la mejora de la percepción de los riesgos, las conductas seguras y la cultura preventiva de todos los trabajadores. Este es el camino hacia el 0 accidentes por elección;

- Percepción del riesgo

- Conducta Preventiva

- Actitud Proactiva

Palabras clave: Conducta segura, actitud preventiva y proactiva, participación de los trabajadores, Curva de Bradley, $O$ accidentes.

\section{Abstract}

Behaviour Based Safety audits system is a preventive tool which helps us to identify unsafe behaviours before the generation of any incident. Thanks to a daily practice at Arcelormittal Tailored Blanks Zaragoza we promote the risk perception improvement, safe behaviours and the preventive culture of all workers. This is the way to 0 accidents... by choice;

- Risk perception

- Preventive behavior

- Proactive approach

Key words: Safe Behaviour, Preventive and Proactive approach, workers participation, Bradley curve, $O$ accidents. 


\section{OBJETIVOS PROPUESTOS}

Desde sus inicios, ArcelorMittal Tailored Blanks Zaragoza ha mantenido un claro compromiso con la seguridad y salud de sus trabajadores, buscando unos índices de accidentabilidad cero gracias a la implicación de todos los trabajadores de la empresa, personal de empresas de trabajo temporal y contratas, de forma que ArcelorMittal Tailored Blanks Zaragoza sea un referente en materia de seguridad y salud tanto a nivel nacional como internacional, tanto dentro como fuera de ArcelorMittal. Nuestros trabajadores se muestran orgullosos de este compromiso y valoran este aspecto de la empresa muy positivamente en las encuestas periódicas de satisfacción del personal.

Una de las herramientas para conseguir estos índices excelentes en materia de prevención de riesgos laborales, son las Revisiones de Conducta basadas en la Seguridad, cuyos objetivos principales son:

- Integración de la seguridad y salud laboral en cada fase del proceso productivo de forma que la seguridad sea la consigna de actuación en todo momento. Esta máxima es recordada todos y cada uno de los días.

- Formación e información continua a todos los trabajadores tanto en materia de seguridad y salud como en aquellos aspectos que pueden afectar a ésta, mediante la charla realizada durante la Revisión de Conducta o posteriormente al detectarse posibles desviaciones debidas a falta de conocimientos u olvidos.

- Adquisición de compromisos por parte de todos los trabajadores, más allá del cumplimiento legal.

- Mejora continua en todas las áreas al identificar pequeñas condiciones y actos inseguros, detectando riesgos antes de que se produzcan los accidentes, reforzando el objetivo de seguir reduciendo el número de incidentes y de mejorar las condiciones de trabajo, buscando puestos de trabajo sin riesgos profesionales, anticipándose a los peligros.



- Involucrar activamente y hacer partícipes a todos y cada uno de los integrantes de la empresa en la cultura preventiva de seguridad y en el concepto de protección proactiva y de equipo, ya que se detectan una serie de incidencias leves repetitivas originadas en conducta.

\section{DESCRIPCIÓN DE LA BUENA PRÁCTICA}

El objetivo buscado con esta Buena Práctica es que todos los trabajadores actúen por sí mismos y reaccionen de inmediato como un equipo, detectando los riesgos y aplicando acciones para evitarlos. Una cultura preventiva excelente e integrada en el trabajo diario permite alcanzar los niveles de equipo proactivo que se detallan en la Curva de Bradley, y que conllevan la eliminación de los accidentes. 


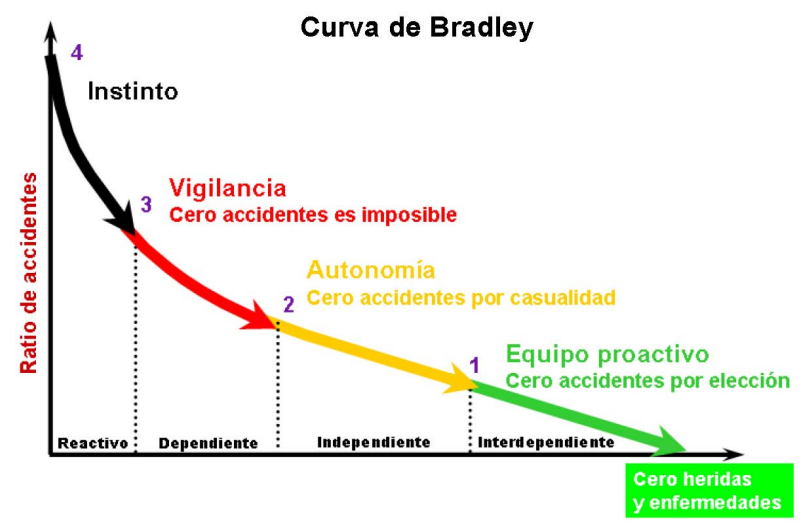

Las Revisiones de Conducta se basan en la observación de una tarea concreta que desarrolla un trabajador. Son realizadas de forma periódica y planificada (por un lado, por parte de dos personas de Oficinas de forma mensual; por otro lado, por una persona de Planta y otra de Oficinas por cada turno de trabajo). Un paso previo a la realización de la Revisión de Conducta es que el equipo de personas que la va a realizar esté formado previamente en la herramienta. La función de cada trabajador es participar de manera proactiva en la detección y comunicación de riesgos, y fomentar conductas seguras entre los propios compañeros de trabajo.

Durante la Revisiones de Conducta y tras un periodo de tiempo de observación de la tarea realizada por el trabajador, los observadores se acercan y le realizan una serie de preguntas al trabajador quedando registrado lo observado en una ficha junto a las propias conclusiones del trabajador y de los observadores al finalizar la revisión. Por norma general, estas preguntas dan origen a debates y/o charlas abiertas en materia de seguridad y salud laboral con el trabajador observado, que conlleva un refuerzo de actitudes positivas, compromisos personales y corrección de conductas inseguras por autoconvencimiento del propio trabajador observado.

Mediante estas charlas, es posible llegar a desvelar algunas situaciones inseguras o actuaciones ocultas o desconocidas que entrañan riesgos no detectados previamente. El objetivo de estas charlas es la concienciación conjunta de los participantes, la confirmación de actitudes proactivas y el refuerzo positivo de los buenos comportamientos. En todo caso, las Revisiones de Conducta no tienen nunca una finalidad sancionadora, aún en el caso de detectarse durante ellas una infracción; su objetivo es constructivo y sirve únicamente para elevar el nivel preventivo de todos los integrantes de la organización.

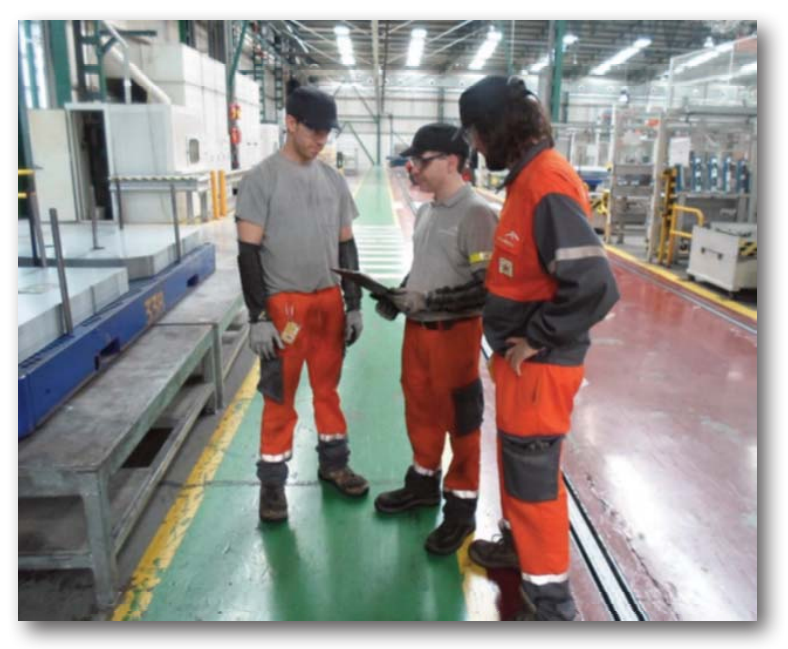

Además, durante las Revisiones de Conducta, también suelen surgir aspectos de organización en las instalaciones, que se canalizan por los sistemas existentes (sugerencias 
de mejora o resolución de etiquetas de seguridad). En caso de un mayor riesgo, el Departamento de Prevención de Riesgos Laborales emite un informe específico y se inicia un plan de acción específico para eliminar o minimizar el riesgo detectado.

Finalmente se realiza un seguimiento de los registros de las Revisiones de Conducta y de las acciones relacionadas mediante la evaluación conjunta de esos registros y el feedback de los participantes. Con esta documentación, el Departamento de Prevención de Riesgos evalúa tendencias, zonas de riesgo, operatividad de las instrucciones de trabajo, etc., según las categorías donde aparecen más desviaciones.

\section{RESULTADOS Y BENEFICIOS OBTENIDOS}

ArcelorMittal Tailored Blanks Zaragoza lleva realizando Revisiones de Conducta desde sus inicios, aunque de manera formal desde el año 2008 con muy buenos resultados. Para este año 2013 hay previsto realizar cerca de 600 Revisiones de Conducta.

Gracias a la participación en esta actividad, se obtiene una mejora propia en cada trabajador, debido al ejercicio de "sentir" la prevención en sus actividades cotidianas, ya que cada trabajador desarrolla una labor continua de mejora y garantía de la seguridad, por medio de su participación directa y periódica en estas Revisiones de Conducta. Además, se produce una satisfacción por ayudar a sus compañeros y se incrementan los niveles de percepción y cultura preventiva propia y del equipo con el que se trabaja, facilitando la comunicación en seguridad y promoviendo y fomentando durante la jornada la comunicación en aspectos de seguridad (buenas prácticas, riesgos detectados, etc.).

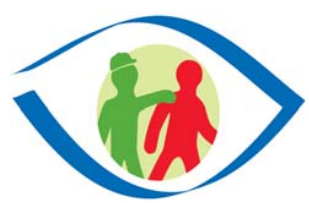

Además de ser un método participativo para mejorar la conducta preventiva de todos los empleados, también es una herramienta de gestión. Es un momento dedicado a conversar sobre seguridad con los trabajadores que ayuda a reconocer buenas prácticas, a identificar puntos débiles en prevención de riesgos laborales y encontrar soluciones, a identificar las normas de prevención que no se cumplen correctamente y a comprender los procedimientos que no son aplicables en la práctica, ya que los trabajadores se cuestionan por qué actúan de una determinada manera y a preocuparse por su entorno.

Desde la puesta en funcionamiento en 2008 se ha conseguido incrementar notablemente el número de anomalías de seguridad detectadas y solucionadas por los propios trabajadores en ese mismo momento, al tiempo que también han aumentado el número de sugerencias de mejora de seguridad propuestas y realizadas por los empleados. Se aprecia claramente una cohesión y mejora de la cultura preventiva de todos los trabajadores.

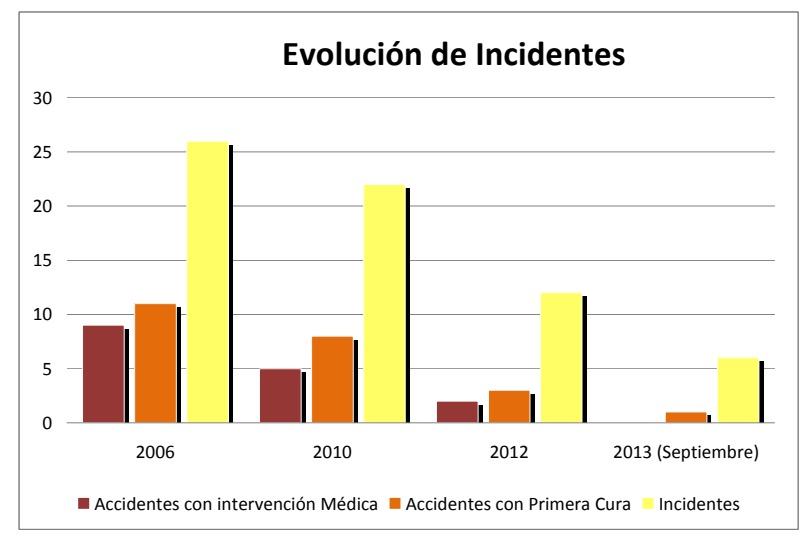




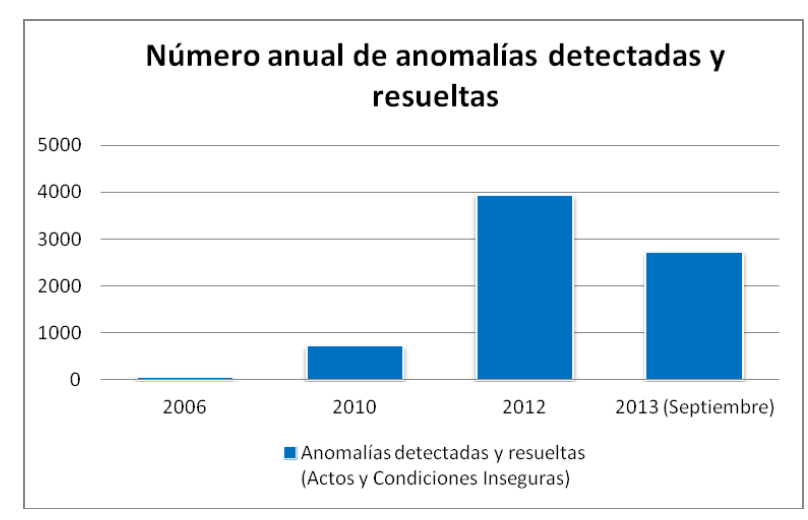

Además, la tendencia en cuanto al número de accidentes e incidentes en los últimos años ha disminuido claramente en relación a los años anteriores, por lo que la participación en la realización de estas Revisiones de Conducta por parte de los trabajadores ha contribuido a aumentar su concienciación en materias de seguridad y salud laboral y una mejora de la conducta individual y grupal respecto a la seguridad.

Todos estos resultados están enfocados hacia el objetivo 0 accidentes, a alcanzar cuando se dispone de un equipo proactivo (Curva de Bradley), y esta herramienta es una palanca importante para esa transformación de los trabajadores.

En resumen, esta herramienta, que puede ser implantada en cualquier tipo de empresa y desarrollada por todos los trabajadores con un mínimo de formación en prevención de riesgos laborales, consiguiendo:

- La integración de todo el personal en materia de seguridad y salud laboral, mediante la integración real de la prevención de riesgos en todos los niveles y trabajadores de la planta.

- La modificación positiva y paulatina de la conducta de todos los trabajadores, obteniéndose unos equipos y personas más concienciados en la seguridad, que ayudar a crear un sentimiento de equipo para trabajar de manera más segura.

- La generación de una reducción de los actos y condiciones inseguras más habituales, al tiempo que se detectan nuevas situaciones, lo cual redunda en medio plazo en una reducción de accidentabilidad y de conductas peligrosas.

- La participación activa de todos los trabajadores en materia de Prevención de Riesgos Laborales.

- La mejora de las condiciones de trabajo, satisfacción y orgullo en los trabajadores por pertenecer y "crear" una empresa segura, lo cual también promueve mejoras en áreas operativas.

- La llamada de atención de un trabajador por otros por detectar que está en una situación de riesgo, es bien recibida, lo que da muestra de que este es el camino hacía el 0 accidentes por elección y enfoca a la mejora.

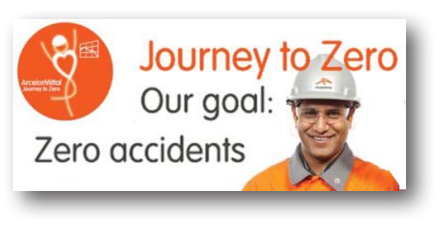

En ArcelorMittal Tailored Blanks Zaragoza queremos mantenernos en el 0 accidentes por elección, y para ello la vigilancia compartida es crucial para minimizar riesgos, ya donde uno no llega, llega un compañero. Y para ello, el $\mathbf{1 0 0} \%$ del personal realiza una actividad en seguridad el $100 \%$ de los días. 